\title{
UMBAUEN IN ZEITEN VON CORONA
}

\section{von Anarchistische Bibliothek | Archiv | Institut für Anarchismusforschung Wien}

Zusammenfassung: Die Anarchistische Bibliothek | Archiv | Institut für Anarchismusforschung Wien übersiedelt mit ihrem gesamten Bestand in ein neues Gebäude, die Räumlichkeiten müssen dazu generalsaniert werden. Der Fokus liegt dabei auf der Verwendung von nachhaltigen Materialien, das Ziel ist eine ökologisch klimaneutrale Bibliothek. Die Renovierungsarbeiten wurden durch Handelsschließungen und personellen Einschränkungen während des COVID-19-bedingten Lockdowns sowie mangelnde finanzielle Mittel erschwert. Der Beitrag zeichnet mittels der laufend als Newsletter verschickten „Baustellen Infos“ die Tätigkeiten und Fortschritte der Anarchistischen Bibliothek während der Monate April bis Oktober 2020 nach.

Schlagwörter: Bibliotheksumbau; Anarchistische Bibliothek Wien; Umzug

\section{RECONSTRUCTION WORK DURING THE CORONA PANDEMIC}

Abstract: The Anarchistische Bibliothek | Archiv | Institut für Anarchismusforschung Wien and its entire library collection will be moving to a new building, the premises will have to be completely renovated before that. The focus is on the use of sustainable materials, the aim being an ecologically climate-neutral library. The renovation work was hampered by closed stores and personnel restrictions during the COVID-19-related lockdown, as well as a lack of financial resources. The article traces the activities and progress of the library during the months of April to October 2020 by means of the "Baustellen Info", which is continuously sent out as a newsletter.

Keywords: library remodeling; Anarchistische Bibliothek Wien; library relocation

DOI: https://doi.org/10.31263/voebm.v73i3-4.5339

(c) Anarchistische Bibliothek | Archiv | Institut für Anarchismusforschung Wien

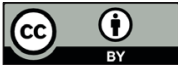

Dieses Werk ist - exkl. einzelner Logos und Abbildungen - lizenziert unter einer Creative-Commons-Lizenz Namensnennung 4.0 International-Lizenz 
Die Anarchistische Bibliothek | Archiv | Institut für Anarchismusforschung Wien (A-bib) gibt es seit 1. Mai 2010. ${ }^{1}$ Also nun über 10 Jahre. Mit Ende März 2019 mussten wir die bisherigen Räumlichkeiten in der Lerchenfelder Straße 124-126 (1080 Wien) verlassen. Das Haus wird generalsaniert und an die Stelle der Bibliothek kommen Garagen. Deshalb waren wir auf der Suche nach neuen Räumlichkeiten. Da wir nicht den rasant steigenden Mietpreisen und der Immobilienspekulation in Wien ausgeliefert sein wollten und ein nachhaltiges und langfristiges Projekt anstreben, haben wir uns entschieden, Räumlichkeiten für Bibliothek und Archiv zu kaufen. Das haben wir geschafft. Der Verein hat ein Lokal im 8. Bezirk in Wien gekauft. Die Räume sind aber renovierungsbedürftig. Da wir sehr beschränkte finanzielle Mittel haben und auch weil wir dem Konzept schöngeistige Arbeiten von handwerklichen Arbeiten zu trennen skeptisch gegenüberstehen, haben wir beschlossen diese Umbauarbeiten, soweit möglich, selbständig durchzuführen. Dies ist ein großes Unterfangen, weil wir uns viele Techniken erst selbständig anlernen mussten, wir nebenher immer wieder neue Gelder für den Umbau aufstellen mussten und auch noch viele - wie könnte es anders sein - bürokratische Hürden zu nehmen hatten. Dass diese Renovierungsarbeiten genau in die Zeit der Corona-Krise gefallen sind, kam noch erschwerend hinzu. Das war auch für uns nicht immer leicht, weil Menschen deshalb teilweise nicht zum Helfen vorbeikommen konnten, weil es bei Bestellungen von Baumaterialien meist große Verzögerungen gab, weil wir selbst einen Umgang finden mussten, wie wir mit der Bedrohung des Virus umgehen. Wir haben uns aber nicht aufhalten lassen und, Lockdown hin oder her, unser Vorhaben weitergeführt und einen Großteil des Umbaus erledigt. Noch sind wir nicht fertig, weil uns noch einige finanzielle Mittel für Licht- und Kücheneinrichtung fehlen. Aber wir hoffen im Frühling mit den Arbeiten abschließen zu können. Womöglich zeitgleich mit der Impfung und der Eindämmung der COVID-Pandemie. Danach wird man uns hoffentlich wieder angstfrei besuchen kommen können, was umso wichtiger sein wird, gilt es ja, nach diesen desaströsen Einschnitt in unser aller Leben eine neue, gerechtere Welt aufzubauen. Um rückblickend einen kleinen Eindruck von diesem Baustellen-Jahr zu gewinnen, findet ihr unser Baustelleninfos, die wir unregelmäßig ausgesendet haben. Es sind sozusagen Einträge aus unserem Baustellen-Logbuch, die verzeichnet haben, wenn wir zwischen den Wirren und Stürmen dieser Bau-Odyssee Zeit dafür gefunden haben.

Viel Vergnügen dabei! 


\section{April 2020: A-bib hat neue Räume!}

Am 1. Mai 2020 hat die Anarchistische Bibliothek | Archiv | Institut für Anarchismusforschung | Wien ihren 10. Geburtstag. Zu diesem Anlass wollen wir euch mitteilen, dass es neue Räume gibt. Diese Räume sind nur ein paar hundert Meter vom alten Standort entfernt. Die A-bib bleibt weiter der Stachel im 8. Bezirk. Vor drei Jahren wurde die „A-BIB BRAUCHT RAUM“-Kampagne gestartet und nun konnten wir schöne Räumlichkeiten kaufen. Danke an alle, welche die Bibliothek unterstützt und mitgeholfen haben. Damit ist der Grundstein für den langfristigen Erhalt der A-Bib gelegt. Aber es gibt noch viel zu tun. Wir haben gerade mit den notwendigen Umbauarbeiten begonnen und dafür braucht es viele Hände, Wissen und Geld. Die Corona-Isolation macht es auch uns nicht gerade leichter und dazu kommen immer wieder Überraschungen auf der Baustelle. Dennoch oder gerade deshalb wollen wir die Bibliothek auch ökologisch nachhaltig gestalten. Wir wollen es - die erste ökologisch klimaneutrale Anarchistische Bibliothek | Archiv | Institut für Anarchismusforschung | Wien. Dafür brauchen wir eure Unterstützung.

Was planen wir also? Um den ökologischen Problemen entgegen zu wirken, und nicht nur davon zu sprechen, machen wir Folgendes:

1. Der Strom, den wir verwenden, kommt zu $100 \%$ aus erneuerbaren Quellen und stammt von mehr als 1.500 Windkraft-, Wasserkraft-, Photovoltaik- und Biogasanlagen aus naher Region.

2. An Gas zum Heizen verwenden wir der Anarchistischen Bibliothek zu $100 \%$ Biogas. Das Biogas kommt aus der nahen Umgebung und ist zu $100 \%$ erneuerbare Energie. Eine angedachte Wärmepumpe ist an diesem Standort zurzeit leider nicht möglich. Windrad und Sonnenenergie sind leider auch nicht möglich, da das Dach mit Luxuswohnungen ausgebaut wurde.

3. Für den neuen Fußboden verwenden wir Linoleum. Linoleum besteht hauptsächlich aus Leinöl, Naturharzen, Kork- oder Holzmehl, Kalksteinpulver, Titanoxid als Weißpigment, Farbstoffen und einem Jutegewebe als Trägerschicht. Unser Linoleum besteht fast vollständig, nämlich zu $98 \%$ aus organischen oder mineralischen Rohstoffen.

4. Die neuen Bücherregale werden aus Birkensperrholz gebaut. Diese Regale wachsen also sozusagen zu $100 \%$ nach. Nicht nur das, auch das $\mathrm{CO}_{2}$ bleibt für Jahrzehnte in den Buchregalen gespeichert.

5. Die Archivregale wachsen zwar nicht nach, aber wurden vor der Zerstörung gerettet und sind somit nachhaltiger als neue Holzregale. 
6. Die barrierefreie Toilette wird zum Teil aus Birkensperrholz und aus hygienischen Gründen mit Melaminharzbeschichtung und Linoleum gemacht. Die Melaminharzbeschichtung ist zwar leider keine Biooberfläche, doch ist uns eine Biooberfläche für diesen sensiblen Bereich in einer langlebigen Qualität nicht bekannt. Auch das eine oder andere Teil aus Metall ist keine nachwachsende Ressource. Doch wir verwenden diese Materialien sparsam, aber auch aus dem Grund, da sie eine sehr lange Lebensdauer aufweisen und es keine Alternativen gibt.

7. Sämtliche Möbel, Türen und Schalldämmungen werden aus nachwachsenden Ressourcen wie Birkensperrholz und Linoleum gestaltet.

8. Die Klimawand wird aus Stampflehm gestampft. Sie sorgt für ein angenehmes Raumklima.

Eine $100 \%$ ige ökologische Nachhaltigkeit ist dabei noch nicht erreicht. Aber zum einen fehlen uns dafür die finanziellen Möglichkeiten (wir bekommen keine Subventionen). Andererseits haben wir weit mehr geschafft, als wir uns es träumen ließen. Natürlich wissen wir, dass viel Kritik an allem möglich wäre - aber anarchistischer als Bakunin, Kropotkin und Malatesta können wir nicht sein - da müssen wir uns eben dahinter anstellen.

\section{Mai 2020}

Nun haben wir auch den Rest des Bauschutts entsorgt - die im Vergleich zur ersten Runde lächerlichen $3 \mathrm{~m}^{3}$ haben wir sorgfältigst getrennt auf die Wiener Müllplätze verteilt. Auf unserer Webseite ${ }^{2}$ seht ihr einige Impressionen von den lieben Holzwürmern, welche Gefallen an den Trägern der Decke gefunden haben und die ihnen sehr zu schmecken scheinen.

Deshalb mussten wir auch die zweite Zwischendecke herausreißen, was eigentlich nicht geplant war und die Folgekosten dafür schon gar nicht. ${ }^{3}$ Aber der erfreuliche Nebeneffekt dabei war, dass ein wunderschönes Gewölbe zum Vorschein kam. Das wird nun verputzt, um die Bücher und Archivmaterialien vor dem langsam herabrieselnden Mauerstaub zu schützen. Die Eisenträger, auf denen das Gewölbe aufliegt, entrosten wir gerade und sie sollen dann mit einer Rostschutzfarbe in Anthrazit gestrichen werden. Wir wollen uns auch bei allen bedanken, welche bereits unser Crowdfunding unterstützt haben. Nach diesem guten Start hoffen wir das die Unterstützung weiter geht. Deshalb bitte die Kampagne fleißig verteilen, wir bauen dafür fleißig weiter. 


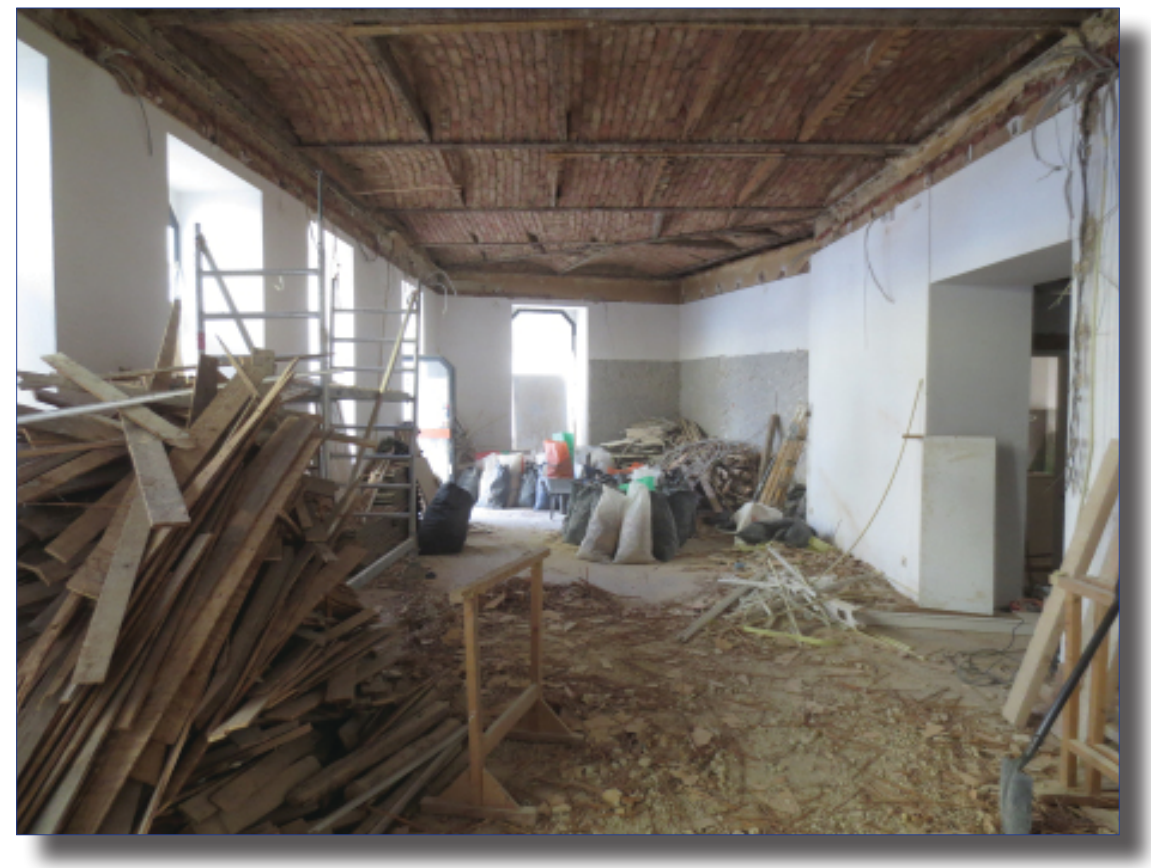

Abb. 1: ??? (Foto: Anarchistische Bibliothek | Archiv | Institut für Anarchismusforschung Wien)

\section{Mai 2020}

Auf dem Gründungskongress der Zweiten Internationale 1889 wurde zum Gedenken an die Opfer des Haymarket Riot der 1. Mai als „Kampftag der Arbeiter*innenbewegung" ausgerufen. Am 1. Mai 1890 wurde zum ersten Mal dieser „Protest- und Gedenktag“ mit Massenstreiks und Massendemonstrationen in der ganzen Welt begangen. 120 Jahre später wurde die A-Bib eröffnet.

Wir haben uns an der antinationalen Banneraktion beteiligt und mitgeholfen den 8. Bezirk zu verschönern. Die Normierungszwänge in unserer Gesellschaft sind echt unglaublich. Wir setzten lieber auf das vernunftbegabte, selbstbestimmte Wesen.

Auch auf der Baustelle ist wieder einiges passiert. Wir haben nun vom Zerstören auf konstruktive Arbeit umgestellt. Das Vergangene musste freilich weg, bevor man das Neue aufbauen kann. Die Eisenträger, denen der Holzwurm nichts anhaben konnte, sind nun mit Rostschutzfarbe gestrichen worden. Die Gastherme ist auch abgebaut, um an diesen Platz Was- 
ser und Abfluss weiter zu verlegen. Wir haben auch den Küchenabfluss gemacht. Nun kommen die Elektroleitungen in der Küche dran, die schon etwas länger darauf warten befestigt zu werden. Das Baugerüst ist in den Kopierraum umgesiedelt, damit wir auch hier die Leitungen verlegen und den I-Träger abschleifen und lackieren können.

Das A-Bib* Crowdfunding läuft seit einigen Tagen - also schaut vorbei und nicht auf das Spenden vergessen. Wir halten euch weiter am Laufenden. Danke an alle die uns bereits unterstützt haben! Und nachdem jetzt alle wieder aus dem Haus gehen dürfen, freuen wir uns über geschickte Hände auf der Baustelle.

\section{Mai 2020}

Die Räume füllen sich schon wieder. Wir haben letzte Woche den Fußboden geliefert bekommen.

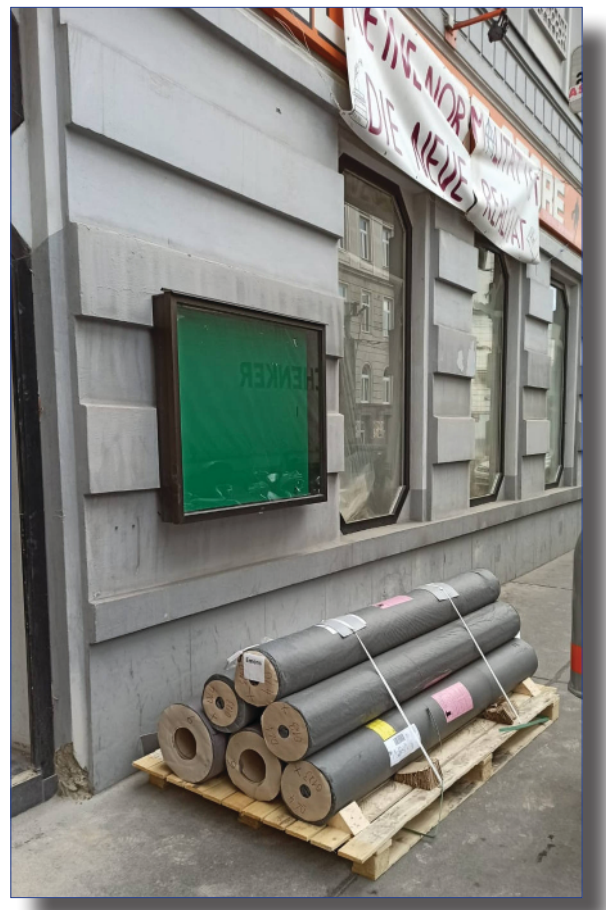

Abb. 2: ??? (Foto: Anarchistische Bibliothek | Archiv | Institut für Anarchismusforschung Wien) 
Es ist ein nachhaltiger Linoleum-Boden, aber dem Gewicht nach zu urteilen, war es ein Betonboden.

Die vergangenen Tage waren wir einkaufen. Das alles soll nun verarbeitet werden und noch viel mehr. Elektroschläuche sind nun dort wo sie sein sollen. Die Wasserleitung für die Küche ist angeschlossen und das Wasser läuft, ohne irgendwo zu tropfen. Dasselbe gilt auch für den Abfluss.

Eine Herausforderung war das Gewölbe im Kopierraum. Da konnte gleich geübt werden für das große Gewölbe im Bibliotheksraum. Das Ergebnis schaut gut aus und so kann demnächst der nächste Raum begonnen werden.

Neues gibt es auch auf unserer Crowdfunding-Seite. Es gibt Goodies, zum Teil von der Bibliothek, zum anderen von Edition AV Verlag und Bahoe Books. Also hinschauen, spenden und Dankeschöns abholen.

\section{Juni 2020}

Wir wollten euch wieder mal über Fortschritte und Neuigkeiten auf der Baustelle informieren. Mittlerweile sind wir dabei die Elektroleitungen zu verlegen, was auch viel Denkarbeit ist. Denn einmal zugemauert lässt sich eine neue Steckdose oder ein vergessener Anschluss nur mehr schwer nachholen. Ein guter Plan ist also die Voraussetzung, um dann trotzdem immer wieder beim Improvisieren zu landen. Da für die meisten Menschen die Quarantänezeiten vorbei sind, kommen auch vermehrt Leute zum Helfen auf die Baustelle, worüber wir uns natürlich sehr freuen. Also falls jemand Lust hat sich einzubringen, schreibt uns einfach eine Mail! Denn schlussendlich ist die A-bib das, was wir daraus machen.

Das A-Bib* Crowdfunding läuft übrigens immer noch und man kann sich schöne, faire, ökologische T-Shirts und guten Lesestoff dabei abholen.

\section{Juni 2020}

Wieder einmal gibt's eine Info über den Fortschritt auf der A-Bib-Baustelle. Diesmal ist es wieder ein großer Schritt. Die Ziegel vom Gewölbe verschwinden hinter dem Putz und die E-Leitungen nehmen auch immer mehr Gestalt an. Es wird aber noch einige Zeit brauchen bis der Strom durch die Aderleitungen fließen kann. Dafür brauchen wir noch etwas Zeit und 
auch noch einige Euros. Schaut auf unser Crowdfunding, da könnt ihr die A-Bib mit kleinen aber auch großen Spenden unterstützen. Das ist natürlich auch auf das Konto der A-Bib möglich. Dabei wollen wir auch gleich an die vielen Menschen, die uns schon unterstützt haben, vielen lieben Dank! sagen. Hier gibt es die neuen Bilder Bilder - Umbau - Baustelle 06 für die, welche sehen wollen was weiter geht.

\section{August 2020}

Nach langem intensiven Arbeiten haben wir nun wieder einen kurzen Baustellenüberblick für euch. Das Crowdfunding ist mit Erfolg abgeschlossen. Und es wurden bereits die Spenden an uns ausbezahlt. An alle Spender*innen ein liebes und anarchistisches Dankeschön!

Die Arbeiten schreiten voran. Nun sind die Mauern in der Bibliothek wieder verputzt sind und das Gewölbe ist auch schon ausgemalt. Echt super. Ein weiteres Danke an die Helfer*innen, denen die Bibliothek es zu verdanken hat in letzter Zeit so weit gekommen zu sein. Die Stromhauptleitung wurde in die Wand verlegt und geht nicht mehr quer durch den Raum.

Ein weiters Projekt der letzten Tage war unser sogenannter „Eiffelturm" - diese Stahlkonstruktion mussten wir für die Verbreiterung des Durchganges zum barrierefreiem WC bauen, damit der Durchgang so breit ist, dass man ohne Probleme mit einem Rollstuhl durchpasst.

Leider haben wir auch nicht unerhebliche laufende Kosten. Jeder Dauerauftrag zur Unterstützung der Bibliothek hilft uns enorm weiter, dass wir auch in dieser Zeit ohne Öffnungszeiten, die Betriebskosten, Strom/Gas, Lagermiete finanzieren können.

\section{August 2020}

Es schreitet voran. Mit der Stampflehmwand haben wir begonnen. Dabei hatte vor Allem die Haltekonstruktion sehr, sehr viel Arbeit bereitet. Die Durchgangskonstruktion „Eiffelturm“ zum WC ist jetzt fix mit dem Mauerwerk verbunden und ergibt eine statische Einheit.

Der Durchbruch ist nun auch gemacht. Damit sich die Träger bei Feuer nicht verdrehen ist eine Brandschutzfarbe angebracht worden. Es geht also immer munter voran. Der neue Stromverteilerkasten ist auch montiert.

Wir wünschen noch allen einen erholsamen coronafreien Urlaub. Wir werden nun auch ein paar Tage versuchen, uns von den Baustrapazen zu erholen. 
Fast hätten wir es vergessen. Wir benötigen noch Spenden für eine Eingangstüre und Fenster. Bei der Eingangstüre kann man leider durchsehen und zwar nicht nur durch die Scheibe, sondern zwischen Türstock und Türrahmen. Bei den Fenstern gibt es das Problem an den Ecken. Die sind gar nicht wärmeisoliert und im Winter wollen wir nicht sinnlos das Biogas, mit dem geheizt wird, verschwenden.

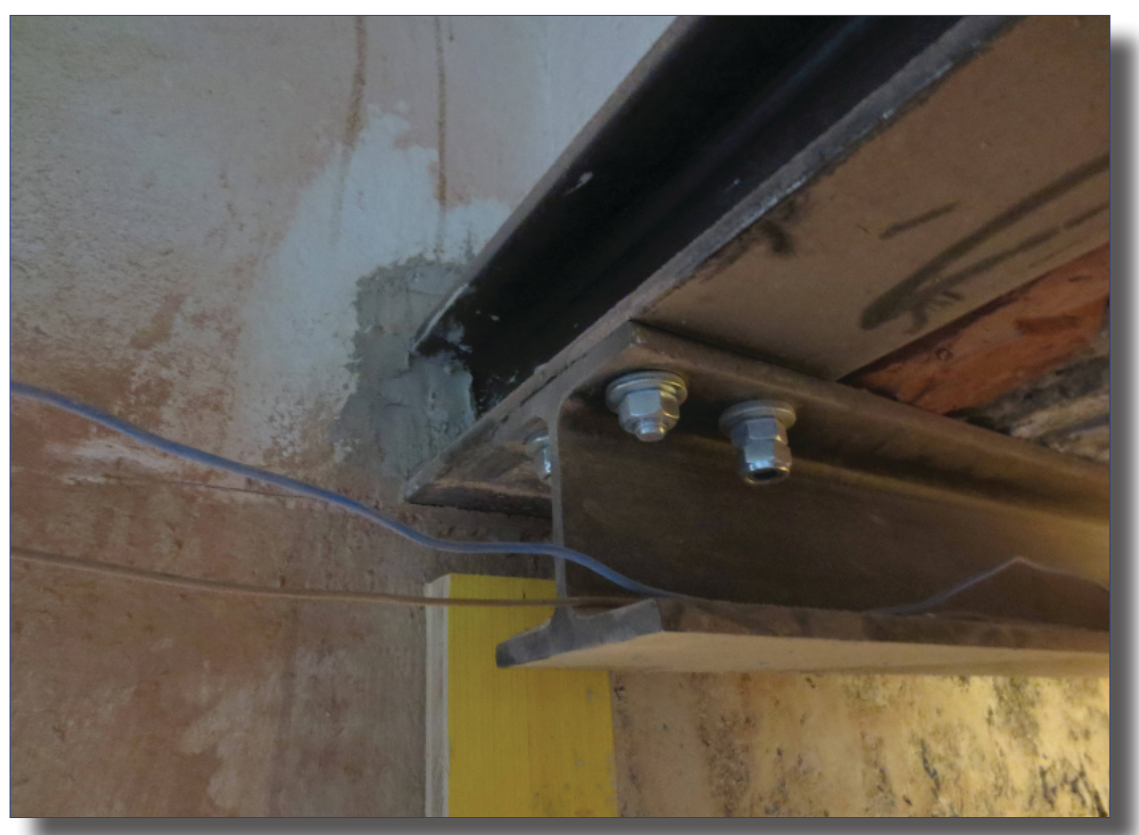

Abb. 3: Durchgangskonstruktion „Eiffelturm“ (Foto: Anarchistische Bibliothek | Archiv | Institut für Anarchismusforschung Wien)

\section{Oktober 2020}

Nach unserer coronafreien Erholung wollen wir euch nun über die jüngsten Baufortschritte informieren.

Die natürliche und nachhaltige Klimaanlage der Anarchistischen Bibliothek, also die Stampflehmwand, ist nun fertig gestampft. Das war echt viel Arbeit. Danke an die Helfer*innen! Nach ein paar Tagen konnten wir sie nun aus ihren Fesseln befreien. Wir haben die Verschalungsplatten entfernt. Nun konnten wir die Lehmwand und ihre Musterung endlich begutachten. Eine sehr schöne bunte Wand kam zum Vorschein. 


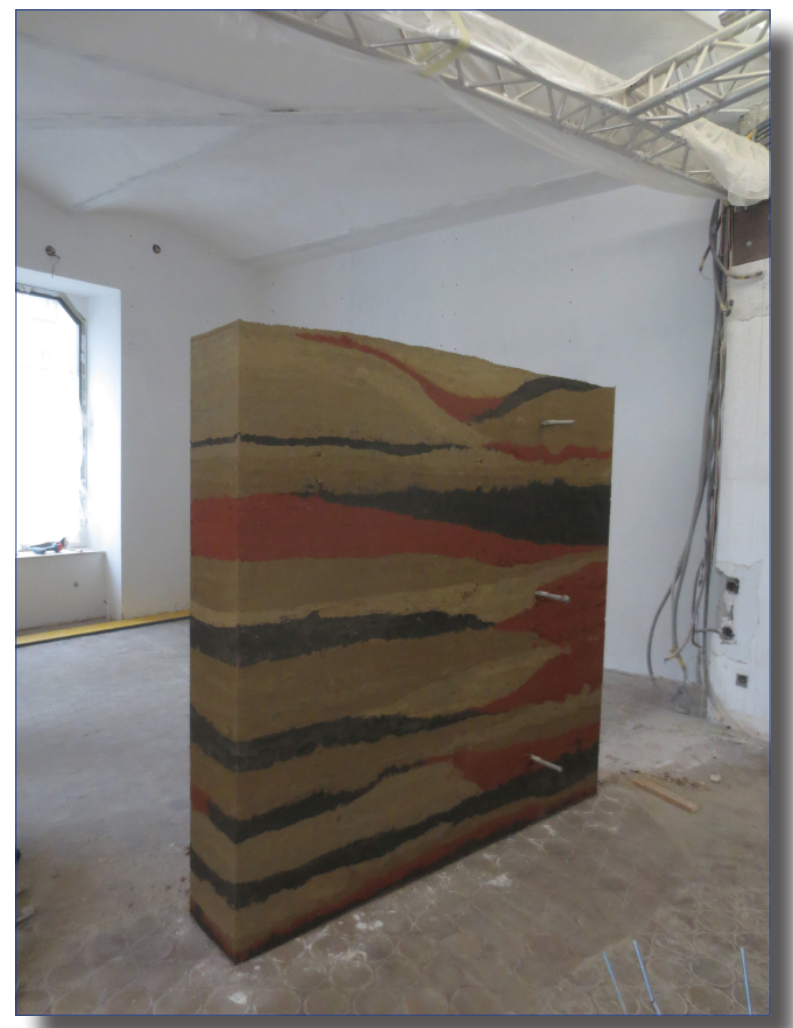

Abb. 4: Die Stampflehmwand, natürliche und nachhaltige Klimaanlage der Anarchistischen Bibliothek (Foto: Anarchistische Bibliothek | Archiv | Institut für Anarchismusforschung Wien)

Da verschiedene Menschen daran gearbeitet haben, ohne sich groß abzusprechen wussten wir alle nicht was uns erwartet. Ein super Ergebnis. Auch konnten wir die Bühnentraversen unterhalb der Decke befestigen. Dort soll die Beleuchtung und verschiedener technische Geräte angebracht werden. Also, es schreitet voran!

Wir dampfen nicht, wir segeln, immer weiter in Richtung Eröffnung unserer neuen Räumlichkeiten. Wer über den neuen Kurs auf dem Laufenden bleiben will, dem sei unsere Internetseite empfohlen: a-bibliothek.org 
Anarchistische Bibliothek | Archiv | Institut für Anarchismusforschung Wien

E-Mail: info@a-bibliothek.org

* Alle Websites und Links wurden zuletzt am 18. Dezember 2020 aufgerufen.

1 Weiterführende Informationen zur Bibliothek: Bibliothekskollektiv Anarchistische Bibliothek \& Archiv Wien (2014). „Anarchistische Bibliothek \& Archiv Wien“. LIBREAS. Library Ideas, 26. https://libreas.eu/ ausgabe26/04kollektiv/

2 Anarchistische Bibliothek \& Archiv I Institut für Anarchismusforschung Wien, Umbau - Baustelle 02: https://a-bibliothek.org/bilder/umbaubaustelle-02/

3 Um die Kosten tragen zu können, wurde auch auf die Methode des (virtuellen) Crowdfundings zurückgegriffen. Unter https://www.startnext. com/a-bib-wien wurde von 15.04. bis 30.06.2020 ein Finanzierungsaufruf gestartet. 